\title{
Sharp boundedness for vector-valued multilinear integral operators
}

Jinsong Pan ${ }^{*}$ and Lijuan Tong

"Correspondence: pjs196855@126.com

Hunan Mechanical and Electrical Polytechnic, Changsha, 410151 P.R. China

\begin{abstract}
In this paper, the sharp inequalities for some vector-valued multilinear integral operators are obtained. As applications, we get the weighted $L^{p}(p>1)$ norm inequalities and an $L \log L$-type estimate for the vector-valued multilinear operators. MSC: 42B20; 42B25

Keywords: vector-valued multilinear operator; Littlewood-Paley operator; Marcinkiewicz operator; Bochner-Riesz operator; sharp estimate; BMO; $A_{p}$-weight
\end{abstract}

\section{Preliminaries and theorems}

As the development of singular integral operators and their commutators, multilinear singular integral operators have been well studied (see [1-5]). In this paper, we study some vector-valued multilinear integral operators as follows.

Suppose that $m_{j}$ are positive integers $(j=1, \ldots, l), m_{1}+\cdots+m_{l}=m$ and $A_{j}$ are functions on $R^{n}(j=1, \ldots, l)$. Let $F_{t}(x, y)$ be defined on $R^{n} \times R^{n} \times[0,+\infty)$. Set

$$
F_{t}(f)(x)=\int_{R^{n}} F_{t}(x, y) f(y) d y
$$

and

$$
F_{t}^{A}(f)(x)=\int_{R^{n}} \frac{\prod_{j=1}^{l} R_{m_{j}+1}\left(A_{j} ; x, y\right)}{|x-y|^{m}} F_{t}(x, y) f(y) d y
$$

for every bounded and compactly supported function $f$, where

$$
R_{m_{j}+1}\left(A_{j} ; x, y\right)=A_{j}(x)-\sum_{|\alpha| \leq m_{j}} \frac{1}{\alpha !} D^{\alpha} A_{j}(y)(x-y)^{\alpha} .
$$

Let $H$ be the Banach space $H=\{h:\|h\|<\infty\}$ such that, for each fixed $x \in R^{n}, F_{t}(f)(x)$ and $F_{t}^{A}(f)(x)$ may be viewed as a mapping from $[0,+\infty)$ to $H$. For $1<s<\infty$, the vector-valued multilinear operator related to $F_{t}$ is defined by

$$
\left|T^{A}(f)(x)\right|_{s}=\left(\sum_{i=1}^{\infty}\left(T^{A}\left(f_{i}\right)(x)\right)^{s}\right)^{1 / s},
$$

(02014 Pan and Tong; licensee Springer. This is an Open Access article distributed under the terms of the Creative Commons Attribution License (http://creativecommons.org/licenses/by/2.0), which permits unrestricted use, distribution, and reproduction in any medium, provided the original work is properly cited. 
where

$$
T^{A}\left(f_{i}\right)(x)=\left\|F_{t}^{A}\left(f_{i}\right)(x)\right\|,
$$

and $F_{t}$ satisfies: for fixed $\varepsilon>0$,

$$
\left\|F_{t}(x, y)\right\| \leq C|x-y|^{-n}
$$

and

$$
\left\|F_{t}(y, x)-F_{t}(z, x)\right\| \leq C|y-z|^{\varepsilon}|x-z|^{-n-\varepsilon}
$$

if $2|y-z| \leq|x-z|$. Set

$$
|T(f)(x)|_{s}=\left(\sum_{i=1}^{\infty}\left|T\left(f_{i}\right)(x)\right|^{s}\right)^{1 / s} \text { and }|f|_{s}=\left(\sum_{i=1}^{\infty}\left|f_{i}(x)\right|^{s}\right)^{1 / s} .
$$

Suppose that $|T|_{s}$ is bounded on $L^{p}\left(R^{n}\right)$ for $1<p<\infty$ and weak $\left(L^{1}, L^{1}\right)$-bounded.

Note that when $m=0, T^{A}$ is just a vector-valued multilinear commutator of $T$ and $A$ (see [6]). While when $m>0, T^{A}$ is a non-trivial generalization of the commutator. It is well known that multilinear operators are of great interest in harmonic analysis and have been studied by many authors (see [1-5]). In [7], Hu and Yang proved a variant sharp estimate for the multilinear singular integral operators. In [6], Pérez and Trujillo-Gonzalez proved a sharp estimate for some multilinear commutator. The main purpose of this paper is to prove a sharp inequality for the vector-valued multilinear integral operators. As applications, we obtain the weighted $L^{p}(p>1)$ norm inequalities and an $L \log L$-type estimate for the vector-valued multilinear operators.

First, let us introduce some notations. Throughout this paper, $Q$ will denote a cube of $R^{n}$ with sides parallel to the axes. For any locally integrable function $f$, the sharp function of $f$ is defined by

$$
f^{\#}(x)=\sup _{x \in Q} \frac{1}{|Q|} \int_{Q}\left|f(y)-f_{Q}\right| d y,
$$

where, and in what follows, $f_{Q}=|Q|^{-1} \int_{Q} f(x) d x$. It is well known that (see [8])

$$
f^{\#}(x) \approx \sup _{x \in Q} \inf _{c \in C} \frac{1}{|Q|} \int_{Q}|f(y)-c| d y .
$$

We say that $f$ belongs to $B M O\left(R^{n}\right)$ if $f^{\#}$ belongs to $L^{\infty}\left(R^{n}\right)$ and $\|f\|_{B M O}=\left\|f^{\#}\right\|_{L^{\infty}}$. For $0<$ $r<\infty$, we denote $f_{r}^{\#}$ by

$$
f_{r}^{\#}(x)=\left[\left(|f|^{r}\right)^{\#}(x)\right]^{1 / r} .
$$

Let $M$ be the Hardy-Littlewood maximal operator, that is, $M(f)(x)=\sup _{x \in Q}|Q|^{-1} \times$ $\int_{Q}|f(y)| d y$. For $k \in N$, we denote by $M^{k}$ the operator $M$ iterated $k$ times, i.e., $M^{1}(f)(x)=$ $M(f)(x)$ and $M^{k}(f)(x)=M\left(M^{k-1}(f)\right)(x)$ for $k \geq 2$. 
Let $\Phi$ be a Young function and $\tilde{\Phi}$ be the complementary associated to $\Phi$, we denote the $\Phi$-average by, for a function $f$,

$$
\|f\|_{\Phi, Q}=\inf \left\{\lambda>0: \frac{1}{|Q|} \int_{Q} \Phi\left(\frac{|f(y)|}{\lambda}\right) d y \leq 1\right\}
$$

and the maximal function associated to $\Phi$ by

$$
M_{\Phi}(f)(x)=\sup _{x \in Q}\|f\|_{\Phi, Q}
$$

The Young functions to be used in this paper are $\Phi(t)=\exp \left(t^{r}\right)-1$ and $\Psi(t)=t \log ^{r}(t+e)$, the corresponding $\Phi$-average and maximal functions are denoted by $\|\cdot\|_{\exp L^{r}, Q}, M_{\exp L^{r}}$ and $\|\cdot\|_{L(\log L)^{r}, Q}, M_{L(\log L)^{r}}$. We have the following inequality, for any $r>0$ and $m \in N$ (see [6])

$$
M(f) \leq M_{L(\log L)^{r}}(f), \quad M_{L(\log L)^{m}}(f) \approx M^{m+1}(f) .
$$

For $r \geq 1$, we denote that

$$
\|b\|_{\operatorname{osc}_{\exp L^{r}}}=\sup _{Q}\left\|b-b_{Q}\right\|_{\exp L^{r}, Q}
$$

the space $\mathrm{Osc}_{\exp L^{r}}$ is defined by

$$
\operatorname{Osc}_{\exp L^{r}}=\left\{b \in L_{\log }^{1}\left(R^{n}\right):\|b\|_{\mathrm{osc}_{\exp L^{r}}}<\infty\right\} .
$$

It has been known that (see [6])

$$
\left\|b-b_{2 Q}\right\|_{\exp L^{r}, 2^{k} Q} \leq C k\|b\|_{\operatorname{Osc}_{\exp L^{r}}} .
$$

It is obvious that $\mathrm{Osc}_{\exp L^{r}}$ coincides with the $B M O$ space if $r=1$, and $\mathrm{Osc}_{\exp L^{r}} \subset B M O$ if $r>1$. We denote the Muckenhoupt weights by $A_{p}$ for $1 \leq p<\infty$ (see [8]).

Now we state our main results as follows.

Theorem 1 Let $1<s<\infty, r_{j} \geq 1$ and $D^{\alpha} A_{j} \in \operatorname{Osc}_{\exp L^{r_{j}}}$ for all $\alpha$ with $|\alpha|=m_{j}$ and $j=1, \ldots, l$. Define $1 / r=1 / r_{1}+\cdots+1 / r_{l}$. Then, for any $0<p<1$, there exists a constant $C>0$ such that for any $f=\left\{f_{i}\right\} \in C_{0}^{\infty}\left(R^{n}\right)$ and $x \in R^{n}$,

$$
\left(\left|T^{A}(f)\right|_{s}\right)_{p}^{\#}(x) \leq C \prod_{j=1}^{l}\left(\sum_{\left|\alpha_{j}\right|=m_{j}}\left\|D^{\alpha_{j}} A_{j}\right\|_{\mathrm{Osc}_{\exp L^{r_{j}}}}\right) M_{L(\log L)^{1 / r}}\left(|f|_{s}\right)(x) .
$$

Theorem 2 Let $1<s<\infty, r_{j} \geq 1$ and $D^{\alpha} A_{j} \in \mathrm{Osc}_{\exp L^{r_{j}}}$ for all $\alpha$ with $|\alpha|=m_{j}$ and $j=$ $1, \ldots, l$.

(1) If $1<p<\infty$ and $w \in A_{p}$, then




(2) If $w \in A_{1}$. Define $1 / r=1 / r_{1}+\cdots+1 / r_{l}$ and $\Phi(t)=t \log ^{1 / r}(t+e)$. Then there exists $a$ constant $C>0$ such that for all $\lambda>0$,

$$
\begin{aligned}
& w\left(\left\{x \in R^{n}:\left|T^{A}(f)(x)\right|_{s}>\lambda\right\}\right) \\
& \quad \leq C \int_{R^{n}} \Phi\left[\lambda^{-1} \prod_{j=1}^{l}\left(\sum_{\left|\alpha_{j}\right|=m_{j}}\left\|D^{\alpha_{j}} A_{j}\right\|_{\mathrm{Osc}_{\exp L^{\prime}}}\right)|f(x)|_{s}\right] w(x) d x .
\end{aligned}
$$

Remark The conditions in Theorems 1 and 2 are satisfied by many operators.

Now we give some examples.

Example 1 Littlewood-Paley operators.

Fix $\varepsilon>0$ and $\mu>(3 n+2) / n$. Let $\psi$ be a fixed function which satisfies the following properties:

(1) $\int_{R^{n}} \psi(x) d x=0$,

(2) $|\psi(x)| \leq C(1+|x|)^{-(n+1)}$

(3) $|\psi(x+y)-\psi(x)| \leq C|y|^{\varepsilon}(1+|x|)^{-(n+1+\varepsilon)}$ when $2|y|<|x|$.

We denote that $\Gamma(x)=\left\{(y, t) \in R_{+}^{n+1}:|x-y|<t\right\}$ and the characteristic function of $\Gamma(x)$ by $\chi_{\Gamma(x)}$. The Littlewood-Paley multilinear operators are defined by

$$
\begin{aligned}
& g_{\psi}^{A}(f)(x)=\left(\int_{0}^{\infty}\left|F_{t}^{A}(f)(x)\right|^{2} \frac{d t}{t}\right)^{1 / 2}, \\
& S_{\psi}^{A}(f)(x)=\left[\iint_{\Gamma(x)}\left|F_{t}^{A}(f)(x, y)\right|^{2} \frac{d y d t}{t^{n+1}}\right]^{1 / 2}
\end{aligned}
$$

and

$$
g_{\mu}^{A}(f)(x)=\left[\iint_{R_{+}^{n+1}}\left(\frac{t}{t+|x-y|}\right)^{n \mu}\left|F_{t}^{A}(f)(x, y)\right|^{2} \frac{d y d t}{t^{n+1}}\right]^{1 / 2},
$$

where

$$
\begin{aligned}
& F_{t}^{A}(f)(x)=\int_{R^{n}} \frac{\prod_{j=1}^{l} R_{m_{j}+1}\left(A_{j} ; x, y\right)}{|x-y|^{m}} \psi_{t}(x-y) f(y) d y, \\
& F_{t}^{A}(f)(x, y)=\int_{R^{n}} \frac{\prod_{j=1}^{l} R_{m_{j}+1}\left(A_{j} ; x, z\right)}{|x-z|^{m}} f(z) \psi_{t}(y-z) d z
\end{aligned}
$$

and $\psi_{t}(x)=t^{-n} \psi(x / t)$ for $t>0$. Set $F_{t}(f)(y)=f * \psi_{t}(y)$. We also define that

$$
\begin{aligned}
& g_{\psi}(f)(x)=\left(\int_{0}^{\infty}\left|F_{t}(f)(x)\right|^{2} \frac{d t}{t}\right)^{1 / 2}, \\
& S_{\psi}(f)(x)=\left(\iint_{\Gamma(x)}\left|F_{t}(f)(y)\right|^{2} \frac{d y d t}{t^{n+1}}\right)^{1 / 2}
\end{aligned}
$$

and

$$
g_{\mu}(f)(x)=\left(\iint_{R_{+}^{n+1}}\left(\frac{t}{t+|x-y|}\right)^{n \mu}\left|F_{t}(f)(y)\right|^{2} \frac{d y d t}{t^{n+1}}\right)^{1 / 2},
$$


which are the Littlewood-Paley operators (see [9]). Let $H$ be the space

$$
H=\left\{h:\|h\|=\left(\int_{0}^{\infty}|h(t)|^{2} d t / t\right)^{1 / 2}<\infty\right\}
$$

or

$$
H=\left\{h:\|h\|=\left(\iint_{R_{+}^{n+1}}|h(y, t)|^{2} d y d t / t^{n+1}\right)^{1 / 2}<\infty\right\} .
$$

Then, for each fixed $x \in R^{n}, F_{t}^{A}(f)(x)$ and $F_{t}^{A}(f)(x, y)$ may be viewed as the mapping from $[0,+\infty)$ to $H$, and it is clear that

$$
\begin{aligned}
& g_{\psi}^{A}(f)(x)=\left\|F_{t}^{A}(f)(x)\right\|, \quad g_{\psi}(f)(x)=\left\|F_{t}(f)(x)\right\|, \\
& S_{\psi}^{A}(f)(x)=\left\|\chi_{\Gamma(x)} F_{t}^{A}(f)(x, y)\right\|, \quad S_{\psi}(f)(x)=\left\|\chi_{\Gamma(x)} F_{t}(f)(y)\right\|
\end{aligned}
$$

and

$$
g_{\mu}^{A}(f)(x)=\left\|\left(\frac{t}{t+|x-y|}\right)^{n \mu / 2} F_{t}^{A}(f)(x, y)\right\|, \quad g_{\mu}(f)(x)=\left\|\left(\frac{t}{t+|x-y|}\right)^{n \mu / 2} F_{t}(f)(y)\right\| .
$$

It is easy to see that $g_{\psi}, S_{\psi}$ and $g_{\mu}$ satisfy the conditions of Theorems 1 and 2 (see [10-12]), thus Theorems 1 and 2 hold for $g_{\psi}^{A}, S_{\psi}^{A}$ and $g_{\mu}^{A}$.

Example 2 Marcinkiewicz operators.

Fix $\lambda>\max (1,2 n /(n+2))$ and $0<\gamma \leq 1$. Let $\Omega$ be homogeneous of degree zero on $R^{n}$ with $\int_{S^{n-1}} \Omega\left(x^{\prime}\right) d \sigma\left(x^{\prime}\right)=0$. Assume that $\Omega \in \operatorname{Lip}_{\gamma}\left(S^{n-1}\right)$. The Marcinkiewicz multilinear operators are defined by

$$
\begin{aligned}
& \mu_{\Omega}^{A}(f)(x)=\left(\int_{0}^{\infty}\left|F_{t}^{A}(f)(x)\right|^{2} \frac{d t}{t^{3}}\right)^{1 / 2}, \\
& \mu_{S}^{A}(f)(x)=\left[\iint_{\Gamma(x)}\left|F_{t}^{A}(f)(x, y)\right|^{2} \frac{d y d t}{t^{n+3}}\right]^{1 / 2}
\end{aligned}
$$

and

$$
\mu_{\lambda}^{A}(f)(x)=\left[\iint_{R_{+}^{n+1}}\left(\frac{t}{t+|x-y|}\right)^{n \lambda}\left|F_{t}^{A}(f)(x, y)\right|^{2} \frac{d y d t}{t^{n+3}}\right]^{1 / 2}
$$

where

$$
F_{t}^{A}(f)(x)=\int_{|x-y| \leq t} \frac{\prod_{j=1}^{l} R_{m_{j}+1}\left(A_{j} ; x, y\right)}{|x-y|^{m}} \frac{\Omega(x-y)}{|x-y|^{n-1}} f(y) d y
$$

and

$$
F_{t}^{A}(f)(x, y)=\int_{|y-z| \leq t} \frac{\prod_{j=1}^{l} R_{m_{j}+1}\left(A_{j} ; y, z\right)}{|y-z|^{m}} \frac{\Omega(y-z)}{|y-z|^{n-1}} f(z) d z .
$$


Set

$$
F_{t}(f)(x)=\int_{|x-y| \leq t} \frac{\Omega(x-y)}{|x-y|^{n-1}} f(y) d y .
$$

We also define that

$$
\begin{aligned}
& \mu_{\Omega}(f)(x)=\left(\int_{0}^{\infty}\left|F_{t}(f)(x)\right|^{2} \frac{d t}{t^{3}}\right)^{1 / 2}, \\
& \mu_{S}(f)(x)=\left(\iint_{\Gamma(x)}\left|F_{t}(f)(y)\right|^{2} \frac{d y d t}{t^{n+3}}\right)^{1 / 2}
\end{aligned}
$$

and

$$
\mu_{\lambda}(f)(x)=\left(\iint_{R_{+}^{n+1}}\left(\frac{t}{t+|x-y|}\right)^{n \lambda}\left|F_{t}(f)(y)\right|^{2} \frac{d y d t}{t^{n+3}}\right)^{1 / 2},
$$

which are the Marcinkiewicz operators (see [13]). Let $H$ be the space

$$
H=\left\{h:\|h\|=\left(\int_{0}^{\infty}|h(t)|^{2} d t / t^{3}\right)^{1 / 2}<\infty\right\}
$$

or

$$
H=\left\{h:\|h\|=\left(\iint_{R_{+}^{n+1}}|h(y, t)|^{2} d y d t / t^{n+3}\right)^{1 / 2}<\infty\right\} .
$$

Then it is clear that

$$
\begin{aligned}
& \mu_{\Omega}^{A}(f)(x)=\left\|F_{t}^{A}(f)(x)\right\|, \quad \mu_{\Omega}(f)(x)=\left\|F_{t}(f)(x)\right\|, \\
& \mu_{S}^{A}(f)(x)=\left\|\chi_{\Gamma(x)} F_{t}^{A}(f)(x, y)\right\|, \quad \mu_{S}(f)(x)=\left\|\chi_{\Gamma(x)} F_{t}(f)(y)\right\|
\end{aligned}
$$

and

$$
\mu_{\lambda}^{A}(f)(x)=\left\|\left(\frac{t}{t+|x-y|}\right)^{n \lambda / 2} F_{t}^{A}(f)(x, y)\right\|, \quad \mu_{\lambda}(f)(x)=\left\|\left(\frac{t}{t+|x-y|}\right)^{n \lambda / 2} F_{t}(f)(y)\right\| .
$$

It is easy to see that $\mu_{\Omega}, \mu_{S}$ and $\mu_{\lambda}$ satisfy the conditions of Theorems 1 and 2 (see [13, 14]), thus Theorems 1 and 2 hold for $\mu_{\Omega}^{A}, \mu_{S}^{A}$ and $\mu_{\lambda}^{A}$.

Example 3 Bochner-Riesz operators.

Let $\delta>(n-1) / 2, B_{t}^{\delta}(\hat{f})(\xi)=\left(1-t^{2}|\xi|^{2}\right)_{+}^{\delta} \hat{f}(\xi)$ and $B_{t}^{\delta}(z)=t^{-n} B^{\delta}(z / t)$ for $t>0$. Set

$$
F_{\delta, t}^{A}(f)(x)=\int_{R^{n}} \frac{\prod_{j=1}^{l} R_{m_{j}+1}\left(A_{j} ; x, y\right)}{|x-y|^{m}} B_{t}^{\delta}(x-y) f(y) d y .
$$

The maximal Bochner-Riesz multilinear operators are defined by

$$
B_{\delta, *}^{A}(f)(x)=\sup _{t>0}\left|B_{\delta, t}^{A}(f)(x)\right| .
$$


We also define that

$$
B_{\delta, *}(f)(x)=\sup _{t>0}\left|B_{t}^{\delta}(f)(x)\right|
$$

which is the maximal Bochner-Riesz operator (see [15]). Let $H$ be the space $H=\{h:\|h\|=$ $\left.\sup _{t>0}|h(t)|<\infty\right\}$, then

$$
B_{\delta, *}^{A}(f)(x)=\left\|B_{\delta, t}^{A}(f)(x)\right\|, \quad B_{*}^{\delta}(f)(x)=\left\|B_{t}^{\delta}(f)(x)\right\| .
$$

It is easy to see that $B_{\delta, *}^{A}$ satisfies the conditions of Theorems 1 and 2 (see [16]), thus Theorems 1 and 2 hold for $B_{\delta, *}^{A}$.

\section{Some lemmas}

We give some preliminary lemmas.

Lemma 1 ([3]) Let $A$ be a function on $R^{n}$ and $D^{\alpha} A \in L^{q}\left(R^{n}\right)$ for all $\alpha$ with $|\alpha|=m$ and some $q>n$. Then

$$
\left|R_{m}(A ; x, y)\right| \leq C|x-y|^{m} \sum_{|\alpha|=m}\left(\frac{1}{|\tilde{Q}(x, y)|} \int_{\tilde{Q}(x, y)}\left|D^{\alpha} A(z)\right|^{q} d z\right)^{1 / q}
$$

where $\tilde{Q}$ is the cube centered at $x$ and having side length $5 \sqrt{n}|x-y|$.

Lemma $2([8, \mathrm{p} .485])$ Let $0<p<q<\infty$ and for any function $f \geq 0$, we define that, for $1 / r=1 / p-1 / q$,

$$
\|f\|_{W L^{q}}=\sup _{\lambda>0} \lambda\left|\left\{x \in R^{n}: f(x)>\lambda\right\}\right|^{1 / q}, \quad N_{p, q}(f)=\sup _{E}\left\|f \chi_{E}\right\|_{L^{p}} /\left\|\chi_{E}\right\|_{L^{r}},
$$

where the sup is taken for all measurable sets $E$ with $0<|E|<\infty$. Then

$$
\|f\|_{W L^{q}} \leq N_{p, q}(f) \leq(q /(q-p))^{1 / p}\|f\|_{W L^{q}} .
$$

Lemma 3 ([6]) Let $r_{j} \geq 1$ for $j=1, \ldots, m$, we denote that $1 / r=1 / r_{1}+\cdots+1 / r_{m}$. Then

$$
\frac{1}{|Q|} \int_{Q}\left|f_{1}(x) \cdots f_{m}(x) g(x)\right| d x \leq\|f\|_{\exp L^{r_{1}, Q}} \cdots\|f\|_{\exp L^{r_{m}, Q}}\|g\|_{L(\log L)^{1 / r}, Q}
$$

\section{Proof of the theorem}

It is only to prove Theorem 1 .

Proof of Theorem 1 It suffices to prove for $f \in C_{0}^{\infty}\left(R^{n}\right)$ and some constant $C_{0}$ that the following inequality holds:

$$
\left(\frac{1}{|Q|} \int_{Q} \|\left. T^{A}(f)(x)\right|_{s}-\left.C_{0}\right|^{p} d x\right)^{1 / p} \leq C \prod_{j=1}^{l}\left(\sum_{\left|\alpha_{j}\right|=m_{j}}\left\|D^{\alpha_{j}} A_{j}\right\|_{\mathrm{Osc}_{\exp L_{j}}{ }^{r_{j}}}\right) M_{L(\log L)^{1 / r}}\left(|f|_{s}\right)(x) .
$$


Pan and Tong Journal of Inequalities and Applications 2014, 2014:315

Page 8 of 13

http://www.journalofinequalitiesandapplications.com/content/2014/1/315

Without loss of generality, we may assume $l=2$. Fix a cube $Q=Q\left(x_{0}, d\right)$ and $\tilde{x} \in Q$. Let $\tilde{Q}=$ $5 \sqrt{n} Q$ and $\tilde{A}_{j}(x)=A_{j}(x)-\sum_{|\alpha|=m} \frac{1}{\alpha !}\left(D^{\alpha} A_{j}\right)_{\tilde{Q}} x^{\alpha}$, then $R_{m}\left(A_{j} ; x, y\right)=R_{m}\left(\tilde{A}_{j} ; x, y\right)$ and $D^{\alpha} \tilde{A}_{j}=$ $D^{\alpha} A_{j}-\left(D^{\alpha} A_{j}\right)_{\tilde{Q}}$ for $|\alpha|=m_{j}$. We split $f=g+h=\left\{g_{i}\right\}+\left\{h_{i}\right\}$ for $g_{i}=f_{i} \chi_{\tilde{Q}}$ and $h_{i}=f_{i} \chi_{R^{n} \backslash \tilde{Q}}$. Write

$$
\begin{aligned}
F_{t}^{A}\left(f_{i}\right)(x)= & \int_{R^{n}} \frac{\prod_{j=1}^{2} R_{m_{j}+1}\left(\tilde{A}_{j} ; x, y\right)}{|x-y|^{m}} F_{t}(x, y) f_{i}(y) d y \\
= & \int_{R^{n}} \frac{\prod_{j=1}^{2} R_{m_{j}+1}\left(\tilde{A}_{j} ; x, y\right)}{|x-y|^{m}} F_{t}(x, y) h_{i}(y) d y+\int_{R^{n}} \frac{\prod_{j=1}^{2} R_{m_{j}}\left(\tilde{A}_{j} ; x, y\right)}{|x-y|^{m}} F_{t}(x, y) g_{i}(y) d y \\
& -\sum_{\left|\alpha_{1}\right|=m_{1}} \frac{1}{\alpha_{1} !} \int_{R^{n}} \frac{R_{m_{2}}\left(\tilde{A}_{2} ; x, y\right)(x-y)^{\alpha_{1}}}{|x-y|^{m}} D^{\alpha_{1}} \tilde{A}_{1}(y) F_{t}(x, y) g_{i}(y) d y \\
& -\sum_{\left|\alpha_{2}\right|=m_{2}} \frac{1}{\alpha_{2} !} \int_{R^{n}} \frac{R_{m_{1}}\left(\tilde{A}_{1} ; x, y\right)(x-y)^{\alpha_{2}}}{|x-y|^{m}} D^{\alpha_{2}} \tilde{A}_{2}(y) F_{t}(x, y) g_{i}(y) d y \\
& +\sum_{\left|\alpha_{1}\right|=m_{1},\left|\alpha_{2}\right|=m_{2}} \frac{1}{\alpha_{1} ! \alpha_{2} !} \int_{R^{n}} \frac{(x-y)^{\alpha_{1}+\alpha_{2}} D^{\alpha_{1}} \tilde{A}_{1}(y) D^{\alpha_{2}} \tilde{A}_{2}(y)}{|x-y|^{m}} F_{t}(x, y) g_{i}(y) d y .
\end{aligned}
$$

Then, by Minkowski's inequality, we have

$$
\begin{aligned}
& {\left[\left.\frac{1}{|Q|} \int_{Q}|| T^{A}(f)(x)\right|_{s}-\left.\left|T^{\tilde{A}}(h)\left(x_{0}\right)\right|_{s}\right|^{p} d x\right]^{1 / p}} \\
& \leq\left[\frac{1}{|Q|} \int_{Q}\left|\left\|F_{t}^{A}(f)(x)\right\|_{s}-\left\|F_{t}^{\tilde{A}}(h)\left(x_{0}\right)\right\|_{s}\right|^{p} d x\right]^{1 / p} \\
& \leq\left[\frac{1}{|Q|} \int_{Q}\left(\sum_{i=1}^{\infty}\left\|F_{t}^{A}\left(f_{i}\right)(x)-F_{t}^{\tilde{A}}\left(h_{i}\right)\left(x_{0}\right)\right\|^{s}\right)^{p / s} d x\right]^{1 / p} \\
& \leq\left[\frac{C}{|Q|} \int_{Q}\left(\sum_{i=1}^{\infty}\left\|\int_{R^{n}} \frac{\prod_{j=1}^{2} R_{m_{j}}\left(\tilde{A}_{j} ; x, y\right)}{|x-y|^{m}} F_{t}(x, y) g_{i}(y) d y\right\|^{s}\right)^{p / s} d x\right]^{1 / p} \\
& +\left[\frac { C } { | Q | } \int _ { Q } \left(\sum_{i=1}^{\infty} \| \sum_{\left|\alpha_{1}\right|=m_{1}} \frac{1}{\alpha_{1} !}\right.\right. \\
& \left.\left.\times \int_{R^{n}} \frac{R_{m_{2}}\left(\tilde{A}_{2} ; x, y\right)(x-y)^{\alpha_{1}}}{|x-y|^{m}} D^{\alpha_{1}} \tilde{A}_{1}(y) F_{t}(x, y) g_{i}(y) d y \|^{s}\right)^{p / s} d x\right]^{1 / p} \\
& +\left[\frac { C } { | Q | } \int _ { Q } \left(\sum_{i=1}^{\infty} \| \sum_{\left|\alpha_{2}\right|=m_{2}} \frac{1}{\alpha_{2} !}\right.\right. \\
& \left.\left.\times \int_{R^{n}} \frac{R_{m_{1}}\left(\tilde{A}_{1} ; x, y\right)(x-y)^{\alpha_{2}}}{|x-y|^{m}} D^{\alpha_{2}} \tilde{A}_{2}(y) F_{t}(x, y) g_{i}(y) d y \|^{s}\right)^{p / s} d x\right]^{1 / p} \\
& +\left[\frac { C } { | Q | } \int _ { Q } \left(\sum_{i=1}^{\infty} \| \sum_{\left|\alpha_{1}\right|=m_{1},\left|\alpha_{2}\right|=m_{2}} \frac{1}{\alpha_{1} ! \alpha_{2} !}\right.\right. \\
& \left.\left.\times \int_{R^{n}} \frac{(x-y)^{\alpha_{1}+\alpha_{2}} D^{\alpha_{1}} \tilde{A}_{1}(y) D^{\alpha_{2}} \tilde{A}_{2}(y)}{|x-y|^{m}} F_{t}(x, y) g_{i}(y) d y \|^{s}\right)^{p / s} d x\right]^{1 / p}
\end{aligned}
$$




$$
\begin{aligned}
& +\left[\frac{C}{|Q|} \int_{Q}\left(\sum_{i=1}^{\infty}\left\|F_{t}^{\tilde{A}}\left(h_{i}\right)(x)-F_{t}^{\tilde{A}}\left(h_{i}\right)\left(x_{0}\right)\right\|^{s}\right)^{p / s} d x\right]^{1 / p} \\
& :=I_{1}+I_{2}+I_{3}+I_{4}+I_{5} .
\end{aligned}
$$

Now, let us estimate $I_{1}, I_{2}, I_{3}, I_{4}$ and $I_{5}$, respectively. First, for $x \in Q$ and $y \in \tilde{Q}$, by Lemma 1 , we get

$$
R_{m_{j}}\left(\tilde{A}_{j} ; x, y\right) \leq C|x-y|^{m_{j}} \sum_{\left|\alpha_{j}\right|=m_{j}}\left\|D^{\alpha_{j}} A_{j}\right\|_{\mathrm{Osc}_{\exp L^{r_{j}}}} .
$$

Thus, by Lemma 2 and the weak type $(1,1)$ of $|T|_{s}$, we obtain

$$
\begin{aligned}
& I_{1} \leq C \prod_{j=1}^{2}\left(\sum_{\left|\alpha_{j}\right|=m_{j}}\left\|D^{\alpha_{j}} A_{j}\right\|_{\mathrm{Osc}_{\exp L^{r_{j}}}}\right)\left(\frac{1}{|Q|} \int_{Q}|T(g)(x)|_{s}^{p} d x\right)^{1 / p} \\
& =C \prod_{j=1}^{2}\left(\sum_{\left|\alpha_{j}\right|=m_{j}}\left\|D^{\alpha_{j}} A_{j}\right\|_{\operatorname{Osc}_{\exp ^{r_{j}}}{ }^{r_{j}}}\right)|Q|^{-1} \frac{\left\||T(g)|_{s} \chi_{Q}\right\|_{L^{p}}}{|Q|^{1 / p-1}} \\
& \leq C \prod_{j=1}^{2}\left(\sum_{\left|\alpha_{j}\right|=m_{j}}\left\|D^{\alpha_{j}} A_{j}\right\|_{\mathrm{Osc}_{\exp L_{j}}^{r_{j}}}\right)|Q|^{-1}\left\||T(g)|_{s}\right\|_{W L^{1}} \\
& \leq C \prod_{j=1}^{2}\left(\sum_{\left|\alpha_{j}\right|=m_{j}}\left\|D^{\alpha_{j}} A_{j}\right\|_{\operatorname{Osc}_{\exp L^{r_{j}}}}\right)|Q|^{-1}\left\||g|_{s}\right\|_{L^{1}} \\
& \leq C \prod_{j=1}^{2}\left(\sum_{\left|\alpha_{j}\right|=m_{j}}\left\|D^{\alpha_{j}} A_{j}\right\|_{\operatorname{Osc}_{\exp L_{j}^{r_{j}}}}\right) M\left(|f|_{s}\right)(\tilde{x}) \\
& \leq C \prod_{j=1}^{2}\left(\sum_{\left|\alpha_{j}\right|=m_{j}}\left\|D^{\alpha_{j}} A_{j}\right\|_{\mathrm{Osc}_{\exp L^{r_{j}}}}\right) M_{L(\log L)^{1 / r}}\left(|f|_{S}\right)(\tilde{x}) .
\end{aligned}
$$

For $I_{2}$, note that $\left\|\chi_{Q}\right\|_{\exp L^{r_{2}, Q}} \leq C$, similar to the proof of $I_{1}$ and by using Lemma 3 , we get

$$
\begin{aligned}
& I_{2} \leq C \sum_{\left|\alpha_{2}\right|=m_{2}}\left\|D^{\alpha_{2}} A_{2}\right\|_{\mathrm{Osc}_{\exp L^{r_{2}}}} \sum_{\left|\alpha_{1}\right|=m_{1}}\left(\frac{1}{|Q|} \int_{R^{n}}\left|T\left(D^{\alpha_{1}} \tilde{A}_{1} g\right)(x)\right|_{s}^{p} d x\right)^{1 / p}
\end{aligned}
$$

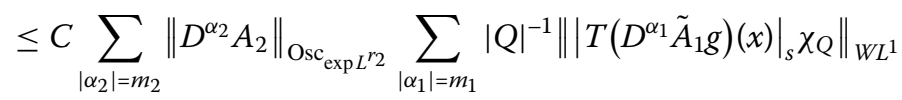

$$
\begin{aligned}
& \leq C \sum_{\left|\alpha_{2}\right|=m_{2}}\left\|D^{\alpha_{2}} A_{2}\right\|_{\mathrm{Osc}_{\mathrm{exp} L^{r_{2}}}} \sum_{\left|\alpha_{1}\right|=m_{1}} \frac{1}{|Q|} \int_{R^{n}}\left|D^{\alpha_{1}} \tilde{A}_{1}(x)\right||g(x)|_{s} d x \\
& \leq C \sum_{\left|\alpha_{2}\right|=m_{2}}\left\|D^{\alpha_{2}} A_{2}\right\|_{\operatorname{Osc}_{\exp L^{r_{2}}}}\left\|\chi_{Q}\right\|_{\exp L^{r_{2}, Q}} \\
& \times \sum_{\left|\alpha_{1}\right|=m_{1}}\left\|D^{\alpha_{1}} A_{1}-\left(D^{\alpha_{1}} A_{1}\right)_{\tilde{Q}}\right\|_{\exp L^{r_{1}, \tilde{Q}}}\left\||f|_{s}\right\|_{L(\log L)^{1 / r}, \tilde{Q}} \\
& \leq C \prod_{j=1}^{2}\left(\sum_{\left|\alpha_{j}\right|=m_{j}}\left\|D^{\alpha_{j}} A_{j}\right\|_{\mathrm{Osc}_{\exp L_{j}} r_{j}}\right) M_{L(\log L)^{1 / r}}\left(|f|_{s}\right)(\tilde{x}) .
\end{aligned}
$$


Pan and Tong Journal of Inequalities and Applications 2014, 2014:315

Page 10 of 13

http://www.journalofinequalitiesandapplications.com/content/2014/1/315

For $I_{3}$, similar to the proof of $I_{2}$, we get

$$
I_{3} \leq C \prod_{j=1}^{2}\left(\sum_{\left|\alpha_{j}\right|=m_{j}}\left\|D^{\alpha_{j}} A_{j}\right\|_{\operatorname{Osc}_{\exp L^{r_{j}}}}\right) M_{L(\log L)^{1 / r}}\left(|f|_{s}\right)(\tilde{x}) .
$$

Similarly, for $I_{4}$, by using Lemma 3 , we get

$$
\begin{aligned}
I_{4} \leq & C \sum_{\left|\alpha_{1}\right|=m_{1},\left|\alpha_{2}\right|=m_{2}}\left(\frac{1}{|Q|} \int_{R^{n}}\left|T\left(D^{\alpha_{1}} \tilde{A}_{1} D^{\alpha_{2}} \tilde{A}_{2} f_{1}\right)(x)\right|_{s}^{p} d x\right)^{1 / p} \\
\leq & C \sum_{\left|\alpha_{1}\right|=m_{1},\left|\alpha_{2}\right|=m_{2}}|Q|^{-1}\left\|\left|T\left(D^{\alpha_{1}} \tilde{A}_{1} D^{\alpha_{2}} \tilde{A}_{2} g\right)\right|_{s} \chi_{Q}\right\|_{W L^{1}} \\
\leq & C \sum_{\left|\alpha_{1}\right|=m_{1},\left|\alpha_{2}\right|=m_{2}} \frac{1}{|Q|} \int_{R^{n}}\left|D^{\alpha_{1}} \tilde{A}_{1}(x) D^{\alpha_{2}} \tilde{A}_{2}(x)\right||g(x)|_{s} d x \\
\leq & C \sum_{\left|\alpha_{1}\right|=m_{1}}\left\|D^{\alpha_{1}} A_{1}-\left(D^{\alpha_{1}} A_{1}\right)_{\tilde{Q}}\right\|_{\exp L^{r_{1}, \tilde{Q}}} \\
& \times \sum_{\left|\alpha_{2}\right|=m_{2}}\left\|D^{\alpha_{2}} A_{2}-\left(D^{\alpha_{2}} A_{2}\right)_{\tilde{Q}}\right\|_{\exp L^{r_{2}, \tilde{Q}} \|}\left\|\left.f\right|_{s}\right\|_{L(\log L)^{1 / r}, \tilde{Q}} \\
\leq & C \prod_{j=1}^{2}\left(\sum_{\left|\alpha_{j}\right|=m_{j}}\left\|D^{\alpha_{j}} A_{j}\right\|_{\operatorname{Osc}_{\exp L_{j}} r_{j}}\right)_{M_{L(\log L)^{1 / r}}\left(|f|_{s}\right)(\tilde{x}) .}
\end{aligned}
$$

For $I_{5}$, we write

$$
\begin{aligned}
& F_{t}^{\tilde{A}}\left(h_{i}\right)(x)-F_{t}^{\tilde{A}}\left(h_{i}\right)\left(x_{0}\right) \\
& =\int_{R^{n}}\left(\frac{F_{t}(x, y)}{|x-y|^{m}}-\frac{F_{t}\left(x_{0}, y\right)}{\left|x_{0}-y\right|^{m}}\right) \prod_{j=1}^{2} R_{m_{j}}\left(\tilde{A}_{j} ; x, y\right) h_{i}(y) d y \\
& +\int_{R^{n}}\left(R_{m_{1}}\left(\tilde{A}_{1} ; x, y\right)-R_{m_{1}}\left(\tilde{A}_{1} ; x_{0}, y\right)\right) \frac{R_{m_{2}}\left(\tilde{A}_{2} ; x, y\right)}{\left|x_{0}-y\right|^{m}} F_{t}\left(x_{0}, y\right) h_{i}(y) d y \\
& +\int_{R^{n}}\left(R_{m_{2}}\left(\tilde{A}_{2} ; x, y\right)-R_{m_{2}}\left(\tilde{A}_{2} ; x_{0}, y\right)\right) \frac{R_{m_{1}}\left(\tilde{A}_{1} ; x_{0}, y\right)}{\left|x_{0}-y\right|^{m}} F_{t}\left(x_{0}, y\right) h_{i}(y) d y \\
& -\sum_{\left|\alpha_{1}\right|=m_{1}} \frac{1}{\alpha_{1} !} \int_{R^{n}}\left[\frac{R_{m_{2}}\left(\tilde{A}_{2} ; x, y\right)(x-y)^{\alpha_{1}}}{|x-y|^{m}} F_{t}(x, y)-\frac{R_{m_{2}}\left(\tilde{A}_{2} ; x_{0}, y\right)\left(x_{0}-y\right)^{\alpha_{1}}}{\left|x_{0}-y\right|^{m}} F_{t}\left(x_{0}, y\right)\right] \\
& \times D^{\alpha_{1}} \tilde{A}_{1}(y) h_{i}(y) d y \\
& -\sum_{\left|\alpha_{2}\right|=m_{2}} \frac{1}{\alpha_{2} !} \int_{R^{n}}\left[\frac{R_{m_{1}}\left(\tilde{A}_{1} ; x, y\right)(x-y)^{\alpha_{2}}}{|x-y|^{m}} F_{t}(x, y)-\frac{R_{m_{1}}\left(\tilde{A}_{1} ; x_{0}, y\right)\left(x_{0}-y\right)^{\alpha_{2}}}{\left|x_{0}-y\right|^{m}} F_{t}\left(x_{0}, y\right)\right] \\
& \times D^{\alpha_{2}} \tilde{A}_{2}(y) h_{i}(y) d y \\
& +\sum_{\left|\alpha_{1}\right|=m_{1},\left|\alpha_{2}\right|=m_{2}} \frac{1}{\alpha_{1} ! \alpha_{2} !} \int_{R^{n}}\left[\frac{(x-y)^{\alpha_{1}+\alpha_{2}}}{|x-y|^{m}} F_{t}(x, y)-\frac{\left(x_{0}-y\right)^{\alpha_{1}+\alpha_{2}}}{\left|x_{0}-y\right|^{m}} F_{t}\left(x_{0}, y\right)\right] \\
& \times D^{\alpha_{1}} \tilde{A}_{1}(y) D^{\alpha_{2}} \tilde{A}_{2}(y) h_{i}(y) d y \\
& =I_{5}^{(1)}+I_{5}^{(2)}+I_{5}^{(3)}+I_{5}^{(4)}+I_{5}^{(5)}+I_{5}^{(6)} \text {. }
\end{aligned}
$$


By Lemma 1 , we know that, for $x \in Q$ and $y \in 2^{k+1} \tilde{Q} \backslash 2^{k} \tilde{Q}$,

$$
\begin{aligned}
\left|R_{m_{j}}\left(\tilde{A}_{j} ; x, y\right)\right| & \leq C|x-y|^{m_{j}} \sum_{\left|\alpha_{j}\right|=m_{j}}\left(\left\|D^{\alpha_{j}} A\right\|_{\mathrm{Osc}_{\exp L} r_{j}}+\left|\left(D^{\alpha_{j}} A\right)_{\tilde{Q}(x, y)}-\left(D^{\alpha_{j}} A\right)_{\tilde{Q}}\right|\right) \\
& \leq C k|x-y|^{m_{j}} \sum_{\left|\alpha_{j}\right|=m_{j}}\left\|D^{\alpha_{j}} A\right\|_{\mathrm{Osc}_{\exp L} r_{j}} .
\end{aligned}
$$

Note that $|x-y| \sim\left|x_{0}-y\right|$ for $x \in Q$ and $y \in R^{n} \backslash \tilde{Q}$, we obtain, by the condition of $F_{t}$,

$$
\begin{aligned}
\left\|I_{5}^{(1)}\right\| \leq & C \int_{R^{n}}\left(\frac{\left|x-x_{0}\right|}{\left|x_{0}-y\right|^{m+n+1}}+\frac{\left|x-x_{0}\right|^{\varepsilon}}{\left|x_{0}-y\right|^{m+n+\varepsilon}}\right) \prod_{j=1}^{2} R_{m_{j}}\left(\tilde{A}_{j} ; x, y\right)\left|h_{i}(y)\right| d y \\
\leq & C \prod_{j=1}^{2}\left(\sum_{\left|\alpha_{j}\right|=m_{j}}\left\|D^{\alpha_{j}} A_{j}\right\|_{\mathrm{Osc}_{\exp L_{j}}{ }^{\prime}}\right) \\
& \times \sum_{k=0}^{\infty} \int_{2^{k+1} \tilde{Q} \backslash 2^{k} \tilde{Q}} k^{2}\left(\frac{\left|x-x_{0}\right|}{\left|x_{0}-y\right|^{n+1}}+\frac{\left|x-x_{0}\right|^{\varepsilon}}{\left|x_{0}-y\right|^{n+\varepsilon}}\right)\left|f_{i}(y)\right| d y \\
\leq & C \prod_{j=1}^{2}\left(\sum_{\left|\alpha_{j}\right|=m_{j}}\left\|D^{\alpha_{j}} A_{j}\right\|_{\operatorname{Osc}_{\exp L_{j}}^{r_{j}}}\right) \sum_{k=1}^{\infty} k^{2}\left(2^{-k}+2^{-\varepsilon k}\right) \frac{1}{\left|2^{k} \tilde{Q}\right|} \int_{2^{k} \tilde{Q}}\left|f_{i}(y)\right| d y .
\end{aligned}
$$

Thus, by Minkowski's inequality, we get

$$
\begin{aligned}
\left(\sum_{i=1}^{\infty}\left\|I_{5}^{(1)}\right\|^{s}\right)^{1 / s} \leq & C \prod_{j=1}^{2}\left(\sum_{|\alpha|=m_{j}}\left\|D^{\alpha} A_{j}\right\|_{\mathrm{Osc}_{\exp L_{j}} r_{j}}\right) \\
& \times \sum_{k=1}^{\infty} k^{2}\left(2^{-k}+2^{-\varepsilon k}\right) \frac{1}{\left|2^{k} \tilde{Q}\right|} \int_{2^{k} \tilde{Q}}|f(y)|_{s} d y \\
\leq & C \prod_{j=1}^{2}\left(\sum_{|\alpha|=m_{j}}\left\|D^{\alpha} A_{j}\right\|_{\operatorname{Osc}_{\exp L} r_{j}}\right) M\left(|f|_{s}\right)(\tilde{x}) \\
\leq & C \prod_{j=1}^{2}\left(\sum_{\left|\alpha_{j}\right|=m_{j}}\left\|D^{\alpha_{j}} A_{j}\right\|_{\operatorname{Osc}_{\exp L_{j}} r_{j}}\right) M_{L(\log L)^{1 / r}\left(|f|_{S}\right)(\tilde{x}) .}
\end{aligned}
$$

For $I_{5}^{(2)}$, by the formula (see [3])

$$
R_{m_{j}}(\tilde{A} ; x, y)-R_{m_{j}}\left(\tilde{A} ; x_{0}, y\right)=\sum_{|\beta|<m_{j}} \frac{1}{\beta !} R_{m_{j}-|\beta|}\left(D^{\beta} \tilde{A} ; x, x_{0}\right)(x-y)^{\beta}
$$

and Lemma 1, we have

$$
\left|R_{m_{j}}(\tilde{A} ; x, y)-R_{m_{j}}\left(\tilde{A} ; x_{0}, y\right)\right| \leq C \sum_{|\beta|<m_{j}} \sum_{|\alpha|=m_{j}}\left|x-x_{0}\right|^{m_{j}-|\beta|}|x-y|^{|\beta|}\left\|D^{\alpha} A\right\|_{\operatorname{Osc}_{\exp L_{j} r_{j}},}
$$


Pan and Tong Journal of Inequalities and Applications 2014, 2014:315

Page 12 of 13

http://www.journalofinequalitiesandapplications.com/content/2014/1/315

thus

$$
\begin{aligned}
\left(\sum_{i=1}^{\infty}\left\|I_{5}^{(2)}\right\|^{s}\right)^{1 / s} & \leq C \prod_{j=1}^{2}\left(\sum_{\left|\alpha_{j}\right|=m_{j}}\left\|D^{\alpha_{j}} A_{j}\right\|_{\operatorname{Osc}_{\exp ^{2} L_{j}}}\right) \sum_{k=0}^{\infty} \int_{2^{k+1} \tilde{Q} \mid 2^{k} \tilde{Q}} k \frac{\left|x-x_{0}\right|}{\left|x_{0}-y\right|^{n+1}}|f(y)|_{s} d y \\
& \leq C \prod_{j=1}^{2}\left(\sum_{\left|\alpha_{j}\right|=m_{j}}\left\|D^{\alpha_{j}} A_{j}\right\|_{\operatorname{Osc}_{\exp L_{j}}}\right) M_{L(\log L)^{1 / r}}\left(|f|_{s}\right)(\tilde{x}) .
\end{aligned}
$$

Similarly,

$$
\left(\sum_{i=1}^{\infty}\left\|I_{5}^{(3)}\right\|^{s}\right)^{1 / s} \leq C \prod_{j=1}^{2}\left(\sum_{\left|\alpha_{j}\right|=m_{j}}\left\|D^{\alpha_{j}} A_{j}\right\|_{\mathrm{Osc}_{\exp L_{j}}{ }^{r_{j}}}\right) M_{L(\log L)^{1 / r}}\left(|f|_{s}\right)(\tilde{x}) .
$$

For $I_{5}^{(4)}$, similar to the proof of $I_{5}^{(1)}, I_{5}^{(2)}$ and $I_{2}$, we get

$$
\begin{aligned}
& \left(\sum_{i=1}^{\infty}\left\|I_{5}^{(4)}\right\|^{s}\right)^{1 / s} \\
& \leq C \sum_{\left|\alpha_{1}\right|=m_{1}} \int_{R^{n} \mid \tilde{Q}}\left\|\frac{(x-y)^{\alpha_{1}} F_{t}(x, y)}{|x-y|^{m}}-\frac{\left(x_{0}-y\right)^{\alpha_{1}} F_{t}\left(x_{0}, y\right)}{\left|x_{0}-y\right|^{m}}\right\| \\
& \times\left|R_{m_{2}}\left(\tilde{A}_{2} ; x, y\right)\right|\left|D^{\alpha_{1}} \tilde{A}_{1}(y)\right||f(y)|_{s} d y \\
& +C \sum_{\left|\alpha_{1}\right|=m_{1}} \int_{R^{n} \backslash \tilde{Q}}\left|R_{m_{2}}\left(\tilde{A}_{2} ; x, y\right)-R_{m_{2}}\left(\tilde{A}_{2} ; x_{0}, y\right)\right| \\
& \times \frac{\left\|\left(x_{0}-y\right)^{\alpha_{1}} F_{t}\left(x_{0}, y\right)\right\|}{\left|x_{0}-y\right|^{m}}\left|D^{\alpha_{1}} \tilde{A}_{1}(y)\right||f(y)|_{s} d y \\
& \leq C \sum_{\left|\alpha_{2}\right|=m_{2}}\left\|D^{\alpha_{2}} A_{2}\right\|_{\mathrm{Osc}_{\exp L^{r_{2}}}} \\
& \times \sum_{\left|\alpha_{1}\right|=m_{1}} \sum_{k=1}^{\infty} k\left(2^{-k}+2^{-\varepsilon k}\right) \frac{1}{\left|2^{k} \tilde{Q}\right|} \int_{2^{k} \tilde{Q}}\left|D^{\alpha_{1}} \tilde{A}_{1}(y)\right||f(y)|_{s} d y \\
& \leq C \sum_{\left|\alpha_{2}\right|=m_{2}}\left\|D^{\alpha_{2}} A_{2}\right\|_{\mathrm{Osc}_{\exp L^{r_{2}}}} \sum_{\left|\alpha_{1}\right|=m_{1}} \sum_{k=1}^{\infty} k\left(2^{-k}+2^{-\varepsilon k}\right) \\
& \times\left\|D^{\alpha_{1}} A_{1}-\left(D^{\alpha_{1}} A_{1}\right)_{\tilde{Q}}\right\|_{\exp L^{r_{1}, 2^{k}} \tilde{Q}}\left\||f|_{s}\right\|_{L(\log L)^{1 / r}, 2^{k} \tilde{Q}} \\
& \leq C \prod_{j=1}^{2}\left(\sum_{\left|\alpha_{j}\right|=m_{j}}\left\|D^{\alpha_{j}} A_{j}\right\|_{\operatorname{Osc}_{\exp L^{r_{j}}}}\right) M_{L(\log L)^{1 / r}}\left(|f|_{s}\right)(\tilde{x}) \text {. }
\end{aligned}
$$

Similarly,

$$
\left(\sum_{i=1}^{\infty}\left\|I_{5}^{(5)}\right\|^{s}\right)^{1 / s} \leq C \prod_{j=1}^{2}\left(\sum_{\left|\alpha_{j}\right|=m_{j}}\left\|D^{\alpha_{j}} A_{j}\right\|_{\operatorname{Osc}_{\exp L^{r_{j}}}}\right) M_{L(\log L)^{1 / r}}\left(|f|_{s}\right)(\tilde{x}) .
$$


For $I_{5}^{(6)}$, by using Lemma 3, we obtain

$$
\begin{aligned}
& \left(\sum_{i=1}^{\infty}\left\|I_{5}^{(6)}\right\|^{s}\right)^{1 / s} \\
& \leq C \sum_{\left|\alpha_{1}\right|=m_{1},\left|\alpha_{2}\right|=m_{2}} \int_{R^{n} \backslash \tilde{Q}}\left\|\frac{(x-y)^{\alpha_{1}+\alpha_{2}} F_{t}(x, y)}{|x-y|^{m}}-\frac{\left(x_{0}-y\right)^{\alpha_{1}+\alpha_{2}} F_{t}\left(x_{0}, y\right)}{\left|x_{0}-y\right|^{m}}\right\| \\
& \quad \times\left|D^{\alpha_{1}} \tilde{A}_{1}(y)\right|\left|D^{\alpha_{2}} \tilde{A}_{2}(y)\right||f(y)|_{s} d y \\
& \leq C \sum_{\left|\alpha_{1}\right|=m_{1},\left|\alpha_{2}\right|=m_{2}} \sum_{k=1}^{\infty}\left(2^{-k}+2^{-\varepsilon k}\right) \frac{1}{\left|2^{k} \tilde{Q}\right|} \int_{2^{k} \tilde{Q}}\left|D^{\alpha_{1}} \tilde{A}_{1}(y)\right|\left|D^{\alpha_{2}} \tilde{A}_{2}(y)\right||f(y)|_{s} d y \\
& \leq C \prod_{j=1}^{2}\left(\sum_{\left|\alpha_{j}\right|=m_{j}}\left\|D^{\alpha_{j}} A_{j}\right\|_{\operatorname{Osc}_{\exp L_{j}} r_{j}}\right) M_{L(\log L)^{1 / r}}\left(|f|_{s}\right)(\tilde{x}) .
\end{aligned}
$$

Thus

$$
I_{5} \leq C \prod_{j=1}^{2}\left(\sum_{\left|\alpha_{j}\right|=m_{j}}\left\|D^{\alpha_{j}} A_{j}\right\|_{\mathrm{Osc}_{\exp L^{r}} r_{j}}\right) M_{L(\log L)^{1 / r}}\left(|f|_{s}\right)(\tilde{x}) .
$$

This completes the proof of Theorem 1 .

By Theorem 1 and the $L^{p}$-boundedness of $M_{L(\log L)^{1 / r}}$, we may obtain the conclusions (1), (2) of Theorem 2.

\section{Competing interests}

The authors declare that they have no competing interests.

\section{Authors' contributions}

The authors completed the paper together. They also read and approved the final manuscript.

Received: 15 January 2014 Accepted: 11 July 2014 Published: 21 Aug 2014

\section{References}

1. Cohen, J: A sharp estimate for a multilinear singular integral on $R^{n}$. Indiana Univ. Math. J. 30,693-702 (1981)

2. Cohen, J, Gosselin, J: On multilinear singular integral operators on $R^{n}$ Stud. Math. 72, 199-223 (1982)

3. Cohen, J, Gosselin, J: A BMO estimate for multilinear singular integral operators. III. J. Math. 30, 445-465 (1986)

4. Coifman, R, Meyer, Y: Wavelets, Calderón-Zygmund and Multilinear Operators. Cambridge Studies in Advanced Math., vol. 48. Cambridge University Press, Cambridge (1997)

5. Ding, Y, Lu, SZ: Weighted boundedness for a class rough multilinear operators. Acta Math. Sin. 17, 517-526 (2001)

6. Pérez, C, Trujillo-Gonzalez, R: Sharp weighted estimates for multilinear commutators. J. Lond. Math. Soc. 65, 672-692 (2002)

7. Hu, G, Yang, DC: A variant sharp estimate for multilinear singular integral operators. Stud. Math. 141, 25-42 (2000)

8. Garcia-Cuerva, J, Rubio de Francia, JL: Weighted Norm Inequalities and Related Topics. North-Holland Math., vol. 116. North-Holland, Amsterdam (1985)

9. Torchinsky, A: Real Variable Methods in Harmonic Analysis. Pure and Applied Math., vol. 123. Academic Press, New York (1986)

10. Liu, LZ: Weighted weak type estimates for commutators of Littlewood-Paley operator. Jpn. J. Math. 29, 1-13 (2003)

11. Liu, LZ: A sharp endpoint estimate for multilinear Littlewood-Paley operator. Georgian Math. J. 11, $361-370$ (2004)

12. Liu, LZ: Sharp endpoint inequality for multilinear Littlewood-Paley operator. Kodai Math. J. 27, 134-143 (2004)

13. Torchinsky, A, Wang, S: A note on the Marcinkiewicz integral. Colloq Math 60(61), 235-243 (1990)

14. Liu, LZ: A sharp estimate for multilinear Marcinkiewicz integral operator. Asian J. Math. 9, 177-184 (2005)

15. Lu, SZ: Four Lectures on Real HP Spaces. World Scientific, River Edge (1995)

16. Wu, B, Liu, LZ: A sharp estimate for multilinear Bochner-Riesz operator. Studia Sci. Math. Hung. 40, 47-59 (2004)

10.1186/1029-242X-2014-315

Cite this article as: Pan and Tong: Sharp boundedness for vector-valued multilinear integral operators. Journal of Inequalities and Applications 2014, 2014:315 